\title{
Poor accuracy of plain radiographic measurements of prosthetic migration and alignment in total ankle replacement
}

\author{
Matthias Braito ${ }^{1}$, Michael Liebensteiner ${ }^{1}$, Dietmar Dammerer ${ }^{1}$, Martin Krismer $^{1}$, Martin Pfurner $^{2}$ \\ and Rainer Biedermann ${ }^{*}$
}

\begin{abstract}
Background: The rotational position of the leg substantially influences measurements in ankle radiographs after total ankle replacement (TAR). The aim of our study was to further specify the influence of different projections on radiographic parameters used to assess component position after TAR.

Methods: The effect of varying degrees of internal rotation, flexion, and ab-/adduction on reference lines in anteroposterior and lateral ankle radiographs was investigated in a cadaveric TAR model. Observed variations were then compared with those found in 34 consecutive patients that received a HINTEGRA total ankle prosthesis in our department.

Results: A change of rotation of $20^{\circ}$ resulted in a variation of measured reference lines of more than $1.3 \mathrm{~cm}$ in anteroposterior radiographs and more than $1 \mathrm{~cm}$ in lateral radiographs in our experimental setting. Even higher intraindividual changes of up to $1.4 \mathrm{~cm}$ were observed in our in vivo series.

Conclusions: The findings suggest that rotational position of the leg highly influences measurements in ankle radiographs after TAR. It further raises the question, if previously described radiographic parameters do provide accurate information for the outcome after TAR in clinical routine as suggested in literature.
\end{abstract}

Keywords: Total ankle replacement, Accuracy, Radiological measurement

\section{Background}

Total ankle replacement (TAR) has become a remarkable treatment option for end-stage osteoarthritis of the ankle joint [1-3], but the potential risks of small improvement of range of motion, persisting pain, and low functional outcome following TAR still remains high $[1,4,5]$. Therefore, hindfoot and total ankle component alignment with regard to biomechanical contact stresses and clinical outcome have been studied extensively [6-13]. Those radiographic parameters used to investigate component malalignment [6, 12-14], subsidence [15, 16], ankle alignment $[3,11,17,18]$, and range of motion $[9,10,19]$ are substantially influenced by the rotational position of the leg while taking the radiographs. The aim of our study

\footnotetext{
* Correspondence: rainer.biedermann@i-med.ac.at

${ }^{1}$ Department of Orthopedics, Medical University of Innsbruck, Anichstrasse

35, 6020 Innsbruck, Austria

Full list of author information is available at the end of the article
}

was to specify the influence of varying radiographic projections of the ankle joint on reference lines and to compare those experimentally observed variations with those observed in in-vivo specimens.

\section{Methods}

In the first step, we conducted an experimental study using a post-mortem ankle specimen to investigate the influence of different radiographic projections on radiographic parameters after TAR. In a second step, observed variations were then compared with the results of radiological measurements of a retrospectively assessed cohort of 34 consecutive patients that received a HINTEGRA total ankle prosthesis (Newdeal, Lyon, France; Integra, Plainsboro, NJ, USA) at our department between 2004 and 2007. The study has been approved by the local ethics committee, and all persons gave their informed consent prior to their inclusion in the study. 


\section{Preparation of specimen in experimental series}

An ankle specimen was obtained by transtibial amputation post-mortem from a male donor without any ankle deformity. The specimen was dissected free of soft tissue, but all major supporting ligaments were kept intact. A HINTEGRA total ankle prosthesis (tibia and talus component size 4, inlay-height $5 \mathrm{~mm}$ ) was implanted into the ankle. The specimen was then fixated on a testing machine (Fig. 1), which allowed rotation along the longitudinal axis of the tibia and flexion/extension at a virtual axis about the knee joint. For lateral radiographs, the testing machine was positioned in a neutral or a slight ab-/adduction position of $5^{\circ}$ to simulate for varus/ valgus malpositioning of the implant.

\section{Measurements in experimental series}

All x-rays were obtained as digital images (Optimus SCP 80, Philipps, Amsterdam, Netherlands). We assumed a rotational variation of $\pm 10^{\circ}$ in real patient's series starting from a neutral position of $10^{\circ}$ internal rotation, which corresponds to the routine position of an ankle joint when taking radiographs at our radiological department. A series of 11 anteroposterior $\mathrm{x}$-rays was obtained from $0^{\circ}$ to $20^{\circ}$ of internal rotation at $0^{\circ}$ of flexion, while the ab-/adduction position was fixed. The same series of $\mathrm{x}$-rays was repeated with $5^{\circ}$ of flexion at a virtual axis about the knee joint to simulate a flexion contracture of the knee joint. Subsequently, three series of lateral $\mathrm{x}$-rays were taken with the shank in $5^{\circ}$ abduction, $0^{\circ}$ ab-/ adduction, and $5^{\circ}$ adduction (Fig. 2) and the same range of rotation as described above to simulate for valgus/ varus positioning. Consequently, we obtained 22 anteroposterior and 33 lateral $\mathrm{x}$-rays in total.

Anteroposterior and lateral reference lines were defined to measure position and migration of the tibial and talar components (Figs. 3 and 4 show examples of specimen in plantar flexion): on the anteroposterior view,

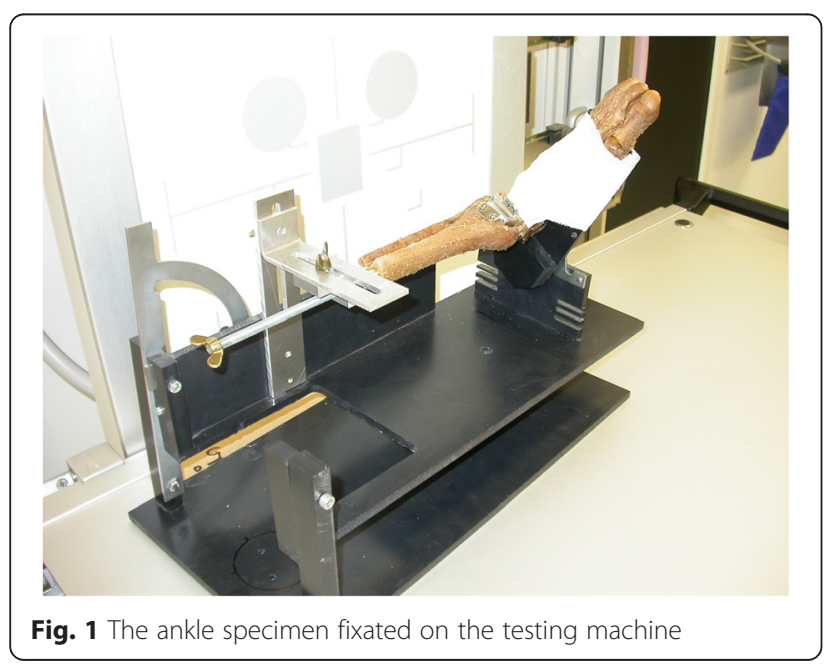

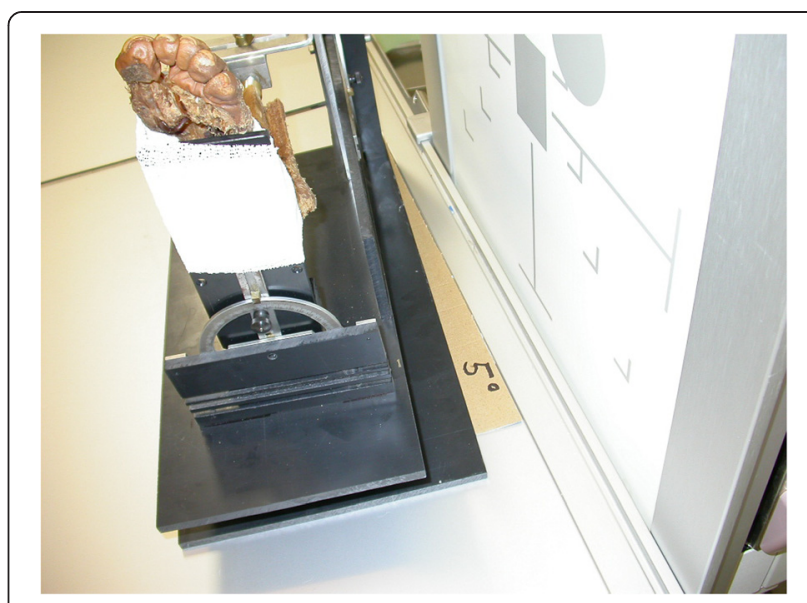

Fig. 2 The testing machine positioned in $5^{\circ}$ of adduction

tibia_vbx1 was defined as the mediolateral distance between the medial border of the tibial component and the lateral border of the medial hole at the shield of the tibial component, tibia_vbx2 as the distance between the lateral border of the tibial component and the medial border of the lateral hole at the shield of the tibial component, tibia_vby as the maximum height of the tibial shield, talus_vbx1 as the length of the talar articulating surface, talus_vbx2 as the distance between the lateral border of the talar component and the lateral border of the talar articulating surface, and talus_vby as the height of the talar component at the articulating surface. On

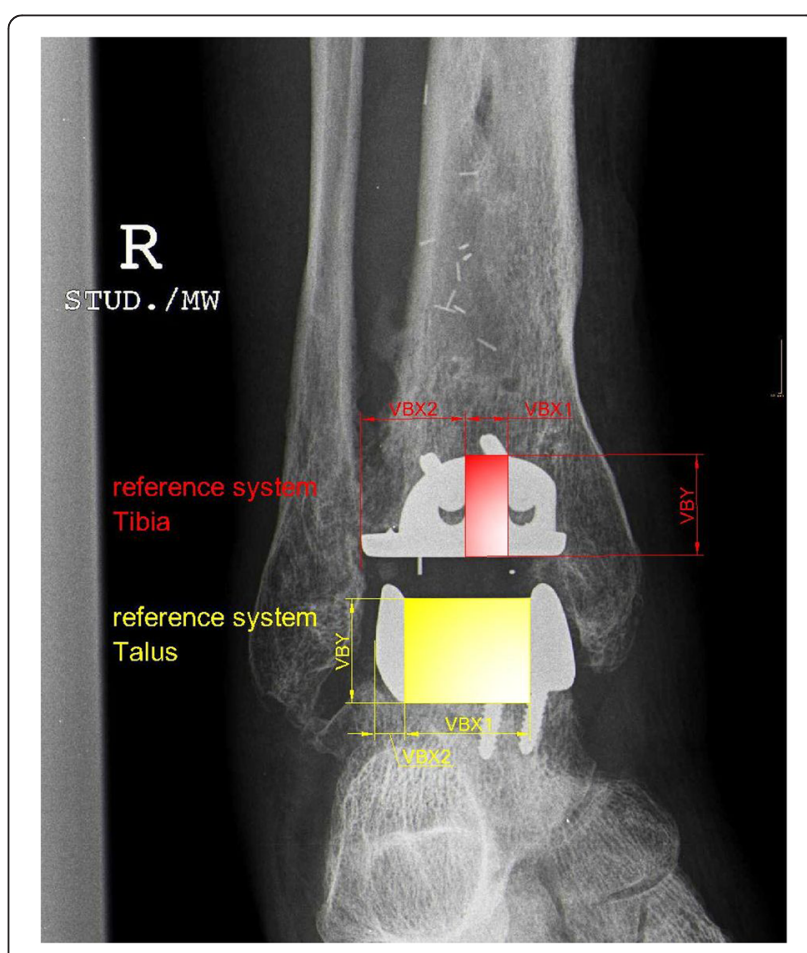

Fig. 3 Anteroposterior reference lines 


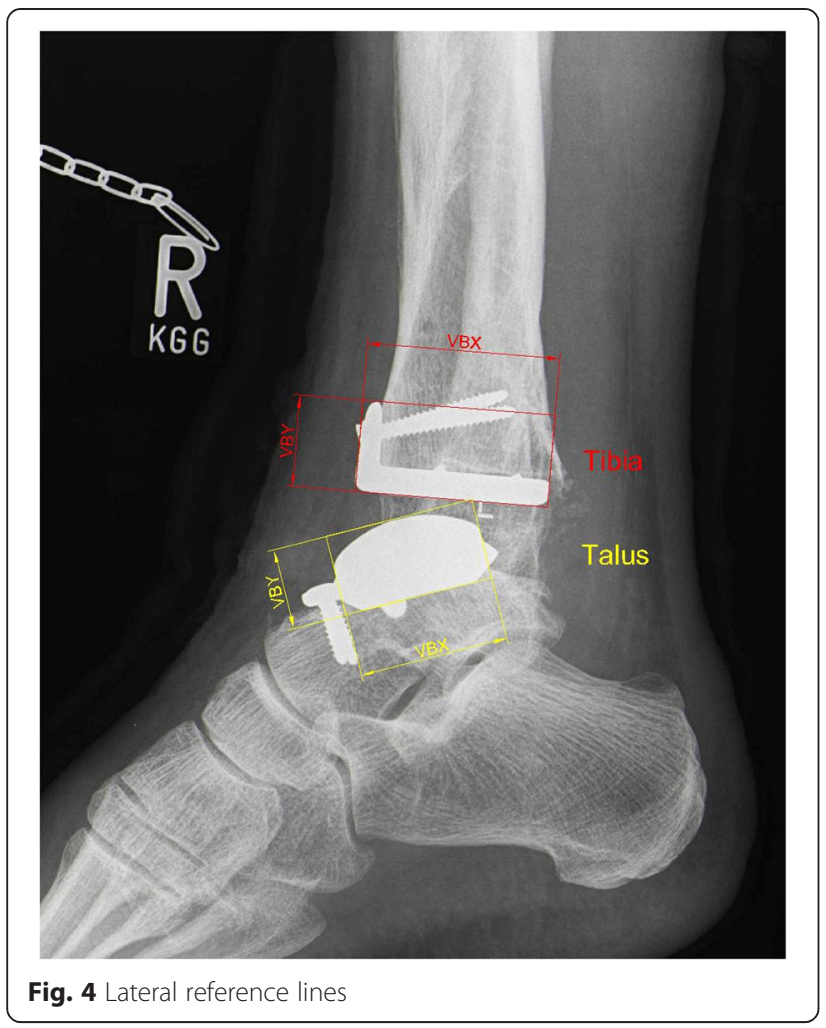

the lateral view, tibia_vbx was defined as the anteroposterior length of the articulating surface of the tibial component, tibia_vby as the height of the tibial shield, talus_vbx as the anteroposterior length of the articulating surface of the talar component, and talus_vby as the height of the talar component (without pegs and screws).

In a pilot study, all measurements were performed by three experienced investigators (M.L., P.M., A.E.) with the use of AutoCAD ${ }^{\circ}$ (Autodesk, Inc., San Francisco, CA, USA) to show the intra- and interobserver reliability. All results were corrected with a magnification factor to correct for specimen-film distance (anteroposterior: magnification factor 1.29 , focus-film distance $110 \mathrm{~cm}$, specimen-film distance $25 \mathrm{~cm}$; lateral: magnification factor 1.15 , focus-film distance $110 \mathrm{~cm}$, specimen-film distance $12 \mathrm{~cm})$.

\section{In-vivo specimens}

In a second step, reference lines were measured in all available standing ankle radiographs (two to eight per patient) taken in clinical routine of 34 consecutive patients that received a HINTEGRA TAR at our department between 2004 and 2007. Results were corrected with an individual magnification factor for focusfilm distance (anteroposterior: magnification factor 0.9, focus-film distance $70 \mathrm{~cm}$, lateral: magnification factor 0.93 , focus-film distance $50 \mathrm{~cm}$ ) and for every size of implant. Data was then compared with the experimental data.

\section{Results}

\section{Experimental series}

Results of measured reference lines measurements in anteroposterior and lateral radiographs are shown in Tables 1 and 2. In the experimental setting, the maximum observed difference was $13.67 \mathrm{~mm}$ (tibia_vbx2) in anteroposterior $\mathrm{x}$-rays and $10.75 \mathrm{~mm}$ (talus_vbx) in lateral $x$-rays. A change of rotation from $0^{\circ}$ to $20^{\circ}$ of internal rotation resulted in a maximum change of $13.02 \mathrm{~mm}$ (tibia_vbx2 at $90^{\circ}$ flexion; Fig. 5) in anteroposterior radiographs and in a maximum change of $6.56 \mathrm{~mm}$ (talus_vbx at $5^{\circ}$ abduction; Fig. 6) in lateral radiographs. Flexion of $5^{\circ}$ about a virtual knee axis resulted in a maximum range of $1.37 \mathrm{~mm}$ (talus_vbx2) in anteroposterior radiographs. A change of ab-/adduction of $10^{\circ}$ (from $5^{\circ}$ of abduction to $5^{\circ}$ of adduction) resulted in a maximum difference of $10.37 \mathrm{~mm}$ (talus_vbx) in lateral radiographs.

\section{In vivo specimens}

Observed differences of measured reference lines in anteroposterior and lateral radiographs are shown in Table 3. The maximum change observed was $13.71 \mathrm{~mm}$ (tibia_vbx2) in anteroposterior $\mathrm{x}$-rays and $14.27 \mathrm{~mm}$ (talus_vbx) in lateral radiographs.

\section{Discussion}

In summary, our study clearly showed that reference lines used for measurement of migration and orientation of components after TAR highly depend on the rotational position of the foot while taking ankle radiographs. A change of internal rotation of $20^{\circ}$ in our experimental series resulted in a maximum change of $1.3 \mathrm{~cm}$ in anteroposterior radiographs and $0.7 \mathrm{~cm}$ in lateral radiographs of the chosen reference lines. We assume difficulties in reproducibly positioning the ankle for a standing radiograph in clinical practice as we found even higher differences of the chosen reference lines in our real patients' series. Therefore, measured variations might be even larger than the value to be determined itself. Assessment of components' migration after TAR might be particularly critical, because a change of prosthesis' position due to different projections may be misinterpreted as a sign of loosening. This might unnecessarily necessitate further investigations such as computed tomography and single photon emission computed tomography scans [20-22] or even revision surgery.

The effect of different rotational projections on consecutive radiological measurements has already been investigated in other orthopedic fields [23-29]. Significant 
Table 1 Observed differences in anteroposterior measurements of experimental series

\begin{tabular}{|c|c|c|c|c|c|c|c|}
\hline & & tibia_vbx1 & tibia_vbx2 & tibia_vby & talus_vbx1 & talus_vbx2 & talus_vby \\
\hline \multirow[t]{4}{*}{$0^{\circ}-20^{\circ}$ internal rotation, $85^{\circ}$ flexion } & Max & 11.42 & 23.32 & 20.25 & 27.41 & 8.39 & 16.44 \\
\hline & Min & 10.79 & 10.64 & 18.85 & 21.60 & 3.41 & 16.13 \\
\hline & Max-min & 0.63 & 12.68 & 1.41 & 5.81 & 4.97 & 0.32 \\
\hline & Mean & 11.11 & 16.54 & 19.72 & 25.18 & 5.23 & 16.22 \\
\hline \multirow[t]{4}{*}{$0^{\circ}-20^{\circ}$ internal rotation, $90^{\circ}$ flexion } & Max & 11.62 & 24.31 & 25.11 & 28.18 & 9.87 & 18.74 \\
\hline & Min & 10.92 & 11.29 & 23.71 & 20.84 & 4.04 & 18.04 \\
\hline & Max-min & 0.70 & 13.02 & 1.40 & 7.34 & 5.83 & 0.70 \\
\hline & Mean & 11.35 & 17.53 & 24.54 & 25.51 & 6.11 & 18.39 \\
\hline \multirow[t]{4}{*}{$85^{\circ}-90^{\circ}$ flexion } & Max & 0.62 & 1.48 & 5.31 & 1.07 & 1.48 & 2.60 \\
\hline & Min & 0.05 & 0.65 & 4.59 & 0.11 & 0.11 & 1.60 \\
\hline & Max-min & 0.57 & 0.83 & 0.72 & 0.97 & 1.37 & 1.01 \\
\hline & Mean & 0.30 & 0.99 & 4.82 & 0.52 & 0.88 & 2.17 \\
\hline \multirow[t]{4}{*}{$0^{\circ}-20^{\circ}$ internal rotation, $85^{\circ}-90^{\circ}$ flexion } & Max & 11.62 & 24.31 & 25.11 & 28.18 & 9.87 & 18.74 \\
\hline & Min & 10.79 & 10.64 & 18.85 & 20.84 & 3.41 & 16.13 \\
\hline & Max-min & 0.82 & 13.67 & 6.26 & 7.34 & 6.46 & 2.61 \\
\hline & Mean & 11.23 & 17.04 & 22.13 & 25.35 & 5.67 & 17.31 \\
\hline
\end{tabular}

changes in the width of the proximal femoral canal with femoral rotation have been reported [23-25]. A shift of the weight-bearing line was described with varying rotation of the foot while taking whole leg radiographs [27] and between supine and standing full-length radiographs
[28]. Moreover, acromial morphology was also found to be dependent on the radiographic projection [29]. In contrast, the effect or different rotational projections in consecutive radiographs after arthroplasty has not specifically addressed so far.

Table 2 Observed differences in lateral measurements of experimental series

\begin{tabular}{|c|c|c|c|c|c|}
\hline & & tibia_vbx & tibia_vby & talus_vbx & talus_vby \\
\hline \multirow[t]{4}{*}{$0^{\circ}-20^{\circ}$ internal rotation, $0^{\circ}$ ab-/adduction } & Max & 48.22 & 20.68 & 39.46 & 22.64 \\
\hline & Min & 45.99 & 17.70 & 34.26 & 17.60 \\
\hline & Max-min & 2.22 & 2.98 & 5.21 & 5.04 \\
\hline & Mean & 46.62 & 19.18 & 36.30 & 20.13 \\
\hline \multirow[t]{4}{*}{$0^{\circ}-20^{\circ}$ internal rotation, $5^{\circ}$ abduction } & Max & 48.82 & 19.31 & 41.30 & 20.55 \\
\hline & Min & 46.42 & 18.38 & 34.73 & 17.83 \\
\hline & Max-min & 2.40 & 0.93 & 6.56 & 2.73 \\
\hline & Mean & 47.11 & 18.79 & 37.49 & 18.67 \\
\hline \multirow[t]{4}{*}{$0^{\circ}-20^{\circ}$ internal rotation, $5^{\circ}$ adduction } & Max & 49.47 & 22.84 & 34.31 & 26.01 \\
\hline & Min & 47.26 & 20.18 & 30.55 & 21.16 \\
\hline & Max-min & 2.20 & 2.66 & 3.77 & 4.85 \\
\hline & Mean & 47.96 & 21.44 & 31.75 & 23.71 \\
\hline \multirow[t]{4}{*}{$5^{\circ}$ abduction $-5^{\circ}$ adduction } & Max & 1.59 & 3.94 & 10.75 & 6.13 \\
\hline & Min & 0.02 & 0.20 & 0.02 & 0.24 \\
\hline & Max-min & 1.57 & 3.73 & 10.73 & 5.88 \\
\hline & Mean & 0.89 & 1.96 & 3.83 & 3.37 \\
\hline \multirow[t]{4}{*}{$0^{\circ}-20^{\circ}$ internal rotation, $5^{\circ}$ abduction $-5^{\circ}$ adduction } & Max & 49.47 & 22.84 & 41.30 & 26.01 \\
\hline & Min & 45.99 & 17.70 & 30.55 & 17.60 \\
\hline & Max-min & 3.47 & 5.14 & 10.75 & 8.40 \\
\hline & Mean & 47.54 & 20.12 & 34.62 & 21.19 \\
\hline
\end{tabular}




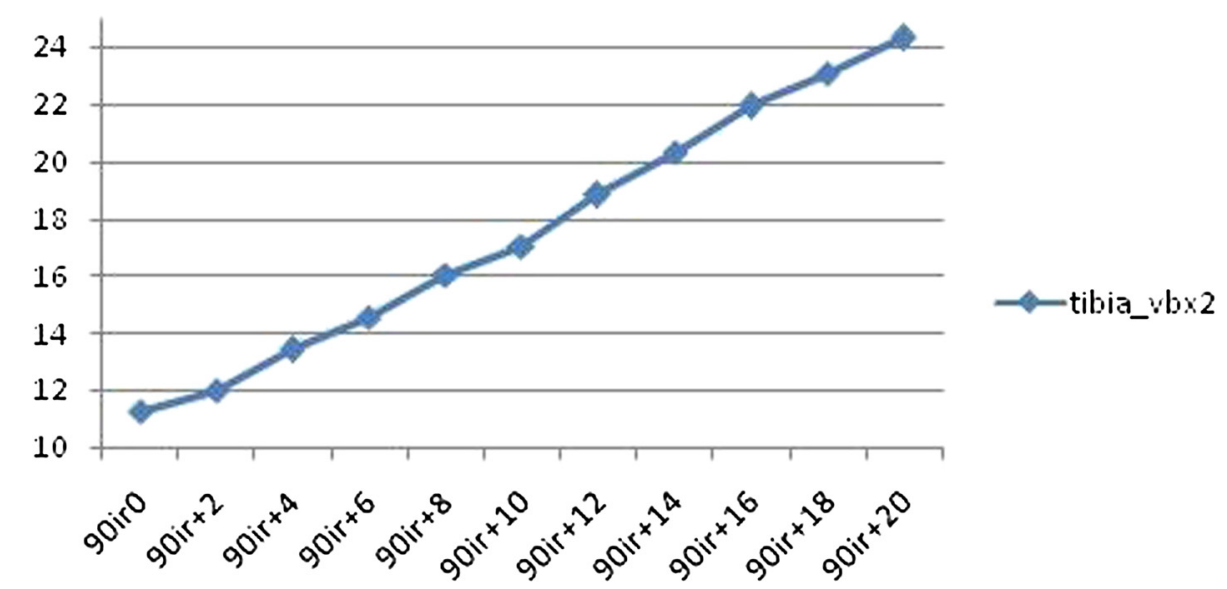

Fig. 5 Change of tibia_vbx2 at $90^{\circ}$ flexion in anteroposterior radiographs

Radiographic measurements further depend on accurate identification of reference points. For our study, we chose simply identifiable reference points on a total ankle prosthesis. The variability of measurements might be even higher using bony landmarks, as they might be blurred and not clearly recognizable with changing rotation or due to periprosthetic ossifications and osteoarthritic changes of adjacent hindfoot joints [30].

On the other hand, most studies dealing with radiographic measurements consider even very small differences in length or degrees of angle as significant $[3,6,12,31-34]$. This raises the question, if radiographic parameters as suggested in literature do provide reliable information on outcome after TAR in clinical routine.

Pyevich et al. [31] evaluated the radiological and clinical outcome of 98 Agility TAR and defined three angular and one linear value to identify migration of the components. Significantly higher rates of pain were found with tibial components placed in more than $4^{\circ}$ of valgus and in 19 cases of migration of prosthetic components. Migration was defined as a change in radiographic position of a component of more than $5^{\circ}$. Reliability of the measurements was tested by "having the same observer [...] repeat the measurements on 60 sets of radiographs with no markings on two separate occasions" and high level of intra-rater reliability was found. However, rotation of the limb was not controlled between serial examinations and accuracy was not tested.

Barg et al. [6] reported a significant superior clinical outcome with respect to pain, AOFAS hindfoot score and ankle ROM in 127 (34.5\%) of 317 osteoarthritic ankles after HINTEGRA TAR with the center of the talar component positioned precisely in the line of the longitudinal axis of the tibia (anteroposterior offset ratio $=0$ ) in lateral ankle radiographs taken with the patient in a standing weight-bearing position. To ensure true lateral radiographs, fluoroscopy was used for all postoperative radiographic examinations. Interobserver and intraobserver reliability was said to be good to excellent for their

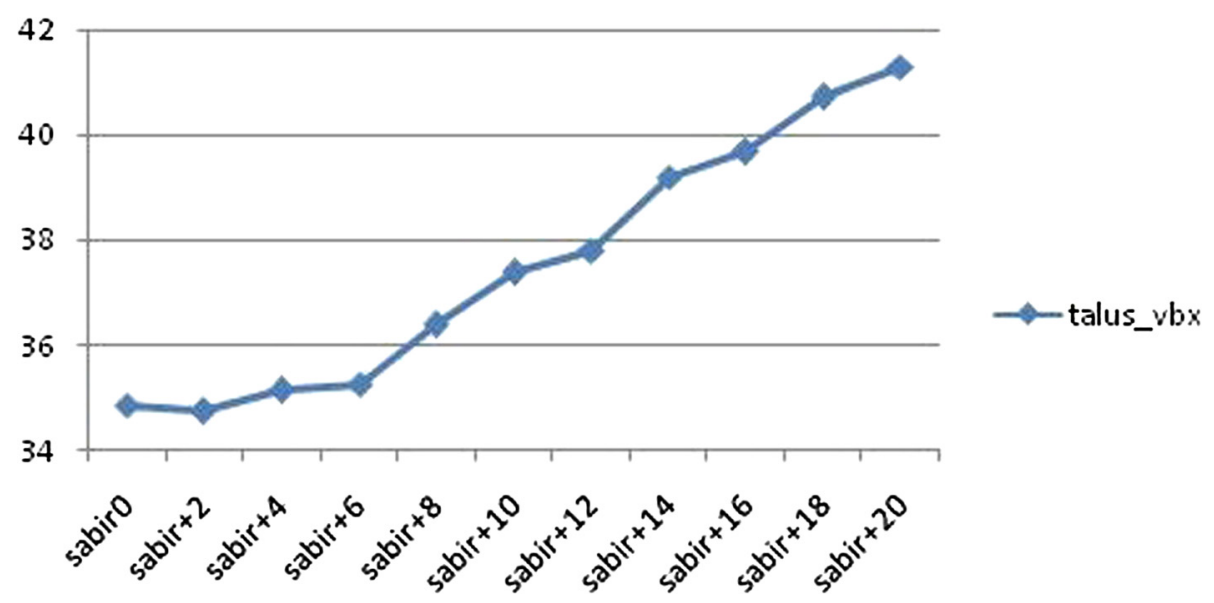

Fig. 6 Change of talus_vbx at $5^{\circ}$ abduction in lateral radiographs 
Table 3 Observed differences in anteroposterior and lateral measurements of in vivo specimens

\begin{tabular}{llllllll}
\hline & & tibia-vbx1 & tibia-vbx2 & tibia-vby & talus-vbx1 & talus-vbx2 & talus-vby \\
\hline Antero-posterior & Max & 2.52 & 13.71 & 7.13 & 11.91 & 9.23 & 6.69 \\
& Mean & 0.81 & 5.12 & 2.64 & 3.75 & 2.99 & 2.35 \\
\hline \multirow{2}{*}{ Lateral } & Max & 10.79 & tibia-vbx & tibia-vby & talus-vbx & talus-vby & \\
& Mean & 4.53 & 1.90 & 14.27 & 7.63 & \\
& & & 4.92 & 2.08 & \\
\hline
\end{tabular}

measurement method. However, no information about accuracy of those measurements is provided.

Hintermann et al. [32] observed migration of two talar components of 122 total ankle replacements measuring the perpendicular distance from the talar component to a line drawn between the dorsal aspect of the talonavicular joint and the calcaneal tubercle. They used fluoroscopy to ensure standardized and true anteroposterior and lateral views of both components. Again, accuracy of these measurements in standard ankle radiographs has not been shown.

Tochigi et al. [33, 34] identified a tibial-axis-to-talus ratio ( $\mathrm{T}-\mathrm{T}$ ratio) as a reliable and valid radiographic measure of anteroposterior tibial-talar alignment that tolerates perturbations of ankle positioning comparably best. They took lateral radiographs of ten cadaver ankles in varying ankle positions in nine prespecified positions in the transverse plane $\left(-20^{\circ}\right.$ to $20^{\circ}$ of internal rotation) and in seven positions in the sagittal plane $\left(-10^{\circ}\right.$ to $20^{\circ}$ of plantar flexion). The low $10^{\circ}$ rotational error in the transversal plane of $2.1 \%$ was explained by the centricity of the chosen anatomical landmarks.

On the basis of these findings, Lee et al. [12] studied the effect of anterior translation of the talus relative to the tibia using the tibiotalar ratio (TTR) and found no difference in clinical outcome after TAR between a group with a TTR within two standard deviations and group with excessive preoperative anterior talar translation (TTR of less than $29 \%$ ). They did not provide information on their used radiographic protocol.

Wood et al. [3] demonstrated that 40 (87\%) of 46 ankles with an anterior translated talus showed an improvement of the TTR after STAR total ankle replacement. The TTR correlated significantly with the inclination of the tibial component. The authors ensured that the ankle was in a similar position on the two radiographs being compared, but no information about accuracy and reliability was provided.

Our data is concordant with those of Tochigi et al. $[33,34]$ and clearly shows that radiographic measurements of prosthetic migration and alignment highly depend on reproducible imaging technique with correct positioning of the foot. However, in their study, Tochigi et al. might have underestimated the variability of the positioning of the foot when taking radiographs in clinical routine as they did not simulated for varying positions of ab-/adduction.

One limitation of the present study is that we were not able to simulate a real ab-/adduction or flexion/ extension of the ankle joint itself due to the rigidity of the obtained ankle specimen. We do not think that this has a big impact on our main message which is the variability of radiographic reference lines. Furthermore, we did not examine the effect of rotation on commonly used markers for alignment of TAR. However, those alignment measurements are underlying a rotational variability to the same extent as the chosen reference lines in our study. Moreover, we did not use a reference ball for calibration, and prosthetic migration/subsidence in our real patient's series might have affected our measurements. Given the short time period between those radiographs, we do not think that this influences our results to a great extent.

\section{Conclusions}

In conclusion, accuracy of most radiographic parameters after TAR suggested in literature has not been tested yet. Our results indicate that measurement errors are likely to be expected. This might lead to wrong interpretation despite a high intra- and interobserver reliability and the use of fluoroscopy. Further studies will have to test for accuracy of suggested radiological parameters, before conclusions about outcome after TAR can be drawn.

\section{Abbreviations}

AOFAS: American Orthopaedic Foot and Ankle Society; Fig.: figure; ROM: range of motion; TAR: total ankle replacement; tibia_vbx1: mediolateral distance between the medial border of the tibial component and the lateral border of the medial hole at the shield of the tibial component (anteroposterior view); tibia_vbx2: distance between the lateral border of the tibial component and the medial border of the lateral hole at the shield of the tibial component (anteroposterior view); tibia_vby: maximum height of the tibial shield (anteroposterior view); talus_vbx1: length of the talar articulating surface (anteroposterior view); talus_vbx2: distance between the lateral border of the talar component and the lateral border of the talar articulating surface (anteroposterior view); talus_vby: height of the talar component at the articulating surface (anteroposterior view); tibia_vbx: anteroposterior length of the articulating surface of the tibial component (lateral view); tibia_vby: height of the tibial shield (lateral view); talus_vbx: anteroposterior length of the articulating surface of the talar component (lateral view); talus_vby: height of the talar component (without pegs and screws lateral view).

\section{Competing interests}

The authors declare that they have no competing interests. 


\section{Authors' contributions}

MB conceived of the study, drafted the manuscript, and performed the statistical analysis. ML carried out the data collection, participated in the study coordination, and helped to draft the manuscript. DD carried out the data collection and participated in the study coordination. MK helped by proofreading the manuscript and revised the manuscript substantially. MP drafted the figures and participated in the data processing. RB helped to draft the manuscript, performed the surgeries, and prepared the ankle specimens. All authors read and approved the final manuscript.

\section{Authors' information}

$\mathrm{RB}$ is a consultant surgeon at the Department of Orthopaedic Surgery Innsbruck and has already conducted a number of studies on outcome after TAR.

\section{Acknowledgements}

We would like to express our very great appreciation to Prof. Peter Mayrhofer for his valuable and constructive contributions in planning and developing this research, Elisabeth Abermann for her support in collecting the data, and Prof. Martin Krismer for his general support of the study.

\section{Author details}

${ }^{1}$ Department of Orthopedics, Medical University of Innsbruck, Anichstrasse 35, 6020 Innsbruck, Austria. ' Geometry and CAD Unit, University of Innsbruck, Innrain 52, 6020 Innsbruck, Austria.

\section{Received: 22 March 2015 Accepted: 10 May 2015}

Published online: 20 May 2015

\section{References}

1. Easley ME, Adams Jr SB, Hembree WC, DeOrio JK. Results of total ankle arthroplasty. J Bone Joint Surg Am. 2011;93(15):1455-68.

2. Barg A, Zwicky L, Knupp M, Henninger HB, Hintermann B. HINTEGRA total ankle replacement: survivorship analysis in 684 patients. J Bone Joint Surg Am. 2013:95(13):1175-83

3. Wood PL, Prem H, Sutton C. Total ankle replacement: medium-term results in 200 Scandinavian total ankle replacements. J Bone Joint Surg (Br). 2008;90(5):605-9.

4. Gougoulias N, Khanna A, Maffulli N. How successful are current ankle replacements? A systematic review of the literature. Clin Orthop Relat Res. 2010;468(1):199-208.

5. Braito M, Dammerer D, Kaufmann G, Fischler S, Carollo J, Reinthaler A, et al. Are our expectations bigger than the results we achieve? A comparative study analysing potential advantages of ankle arthroplasty over arthrodesis. Int Orthop. 2014;38(8):1647-53.

6. Barg A, Elsner A, Anderson AE, Hintermann B. The effect of three-component total ankle replacement malalignment on clinical outcome: pain relief and functional outcome in 317 consecutive patients. J Bone Joint Surg Am. 2011;93(21):1969-78

7. Espinosa N, Walti M, Favre P, Snedeker JG. Misalignment of total ankle components can induce high joint contact pressures. J Bone Joint Surg Am. 2010;92(5):1179-87.

8. Fukuda T, Haddad SL, Ren Y, Zhang LQ. Impact of talar component rotation on contact pressure after total ankle arthroplasty: a cadaveric study. Foot Ankle Int. 2010:31(5):404-11.

9. Tochigi Y, Rudert MJ, Brown TD, Mclff TE, Saltzman CL. The effect of accuracy of implantation on range of movement of the Scandinavian total ankle replacement. J Bone Joint Surg (Br). 2005;87(5):736-40.

10. Cenni F, Leardini A, Cheli A, Catani F, Belvedere C, Romagnoli M, et al. Position of the prosthesis components in total ankle replacement and the effect on motion at the replaced joint. Int Orthop. 2012;36(3):571-8.

11. Haskell A, Mann RA. Ankle arthroplasty with preoperative coronal plane deformity: short-term results. Clin Orthop Relat Res. 2004;424:98-103.

12. Lee KB, Kim MS, Park KS, Cho KJ, Primadhi A. Effect of anterior translation of the talus on outcomes of three-component total ankle arthroplasty. BMC Musculoskelet Disord. 2013;14:260

13. Saltzman CL, Tochigi Y, Rudert MJ, Mclff TE, Brown TD. The effect of agility ankle prosthesis misalignment on the peri-ankle ligaments. Clin Orthop Relat Res. 2004:424:137-42.
14. Rippstein PF, Huber M, Coetzee JC, Naal FD. Total ankle replacement with use of a new three-component implant. J Bone Joint Surg Am. 2011;93(15):1426-35.

15. Anderson T, Montgomery F, Carlsson A. Uncemented STAR total ankle prostheses. Three to eight-year follow-up of fifty-one consecutive ankles. J Bone Joint Surg Am. 2003;85-A(7):1321-9.

16. Kopp FJ, Patel MM, Deland JT, O'Malley MJ. Total ankle arthroplasty with the Agility prosthesis: clinical and radiographic evaluation. Foot Ankle Int. 2006;27(2):97-103.

17. Doets HC, Brand R, Nelissen RG. Total ankle arthroplasty in inflammatory joint disease with use of two mobile-bearing designs. J Bone Joint Surg Am. 2006;88(6):1272-84.

18. Hobson SA, Karantana A, Dhar S. Total ankle replacement in patients with significant pre-operative deformity of the hindfoot. J Bone Joint Surg (Br). 2009;91(4):481-6.

19. Coetzee JC, Castro MD. Accurate measurement of ankle range of motion after total ankle arthroplasty. Clin Orthop Relat Res. 2004;424:27-31.

20. Mason LW, Wyatt J, Butcher C, Wieshmann H, Molloy AP. Single-photonemission computed tomography in painful total ankle replacements. Foot Ankle Int. 2015 Feb 23. [Epub ahead of print].

21. Kohonen IA, Koivu H, Pudas T, Tiusanen H, Vahlberg T, Mattila K. Does computed tomography add information on radiographic analysis in detecting periprosthetic osteolysis after total ankle arthroplasty? Foot Ankle Int. 2013:34(2):180-8

22. Hanna RS, Haddad SL, Lazarus ML. Evaluation of periprosthetic lucency after total ankle arthroplasty: helical CT versus conventional radiography. Foot Ankle Int. 2007;28(8):921-6.

23. Eckrich SG, Noble PC, Tullos HS. Effect of rotation on the radiographic appearance of the femoral canal. J Arthroplasty. 1994;9(4):419-26.

24. Goodman S, Rubenstein J, Schatzker J, Kadish L, Fornasier V. Apparent changes in the alignment of the femoral component in hip arthroplasties associated with limb positioning. Clin Orthop Relat Res. 1987;221:242-5.

25. Albert TJ, Sharkey PF, Chao W, Hume EL, Rothman RH. Rotation affects apparent radiographic positioning of femoral components in total hip arthroplasty. J Arthroplasty. 1991;6(Suppl):S67-71.

26. Ivarsson I, Myrnerts R. The effect of the radiographic projection on the measured position of the prosthesis at knee arthroplasty. Arch Orthop Trauma Surg. 1986;105(6):350-2.

27. Lee YS, Lee BK, Lee SH, Park HG, Jun DS, do Moon H. Effect of foot rotation on the mechanical axis and correlation between knee and whole leg radiographs. Knee Surg Sports Traumatol Arthrosc. 2013:21(11):2542-7.

28. Sabharwal S, Zhao C. Assessment of lower limb alignment: supine fluoroscopy compared with a standing full-length radiograph. J Bone Joint Surg Am. 2008;90(1):43-51.

29. Stehle J, Moore SM, Alaseirlis DA, Debski RE, McMahon PJ. Acromial morphology: effects of suboptimal radiographs. J Shoulder Elbow Surg. 2007;16(2):135-42.

30. Biedermann R, Stockl B, Krismer M, Mayrhofer P, Ornstein E, Franzen H. Evaluation of accuracy and precision of bone markers for the measurement of migration of hip prostheses. A comparison of conventional measurements. J Bone Joint Surg Br. 2001;83(5):767-71.

31. Pyevich MT, Saltzman CL, Callaghan JJ, Alvine FG. Total ankle arthroplasty: a unique design. 2 to 12-year follow-up. J Bone Joint Surg Am. 1998;80(10):1410-20

32. Hintermann B, Valderrabano V , Dereymaeker G, Dick W. The HINTEGRA ankle: rationale and short-term results of 122 consecutive ankles. Clin Orthop Relat Res. 2004:424:57-68.

33. Tochigi Y, Suh JS, Amendola A, Pedersen DR, Saltzman CL. Ankle alignment on lateral radiographs. Part 1: sensitivity of measures to perturbations of ankle positioning. Foot Ankle Int. 2006;27(2):82-7.

34. Tochigi Y, Suh JS, Amendola A, Saltzman CL. Ankle alignment on lateral radiographs. Part 2: reliability and validity of measures. Foot Ankle Int 2006;27(2):88-92 